\title{
Recumbent cycling with integrated volitional control electrical stimulation improves gait speed during the recovery stage in stroke patients
}

\author{
Takuya lyanaga ${ }^{1,2}$, Hayata Abe ${ }^{1,2}$, Takashi Oka ${ }^{1,2}$, Tetsuya Miura ${ }^{3}$, Rumiko Iwasaki ${ }^{1,2}$, Mai Takase ${ }^{1,2}$, Minoru Isatake ${ }^{1,2}$, Atsushi Doi ${ }^{4,5, *}$ \\ 'Department of Rehabilitation, Fukuoka Seisyukai Hospital, Fukuoka, Japan \\ ${ }^{2}$ Center of Advanced Rehabilitation "HOPE", Fukuoka Seisyukai Hospital, Fukuoka, Japan \\ ${ }^{3}$ Department of Rehabilitation, Tsutsumi Hospital, Fukuoka, Japan \\ ${ }^{4}$ Department of Rehabilitation, Faculty of Health Science, Kumamoto Health Science University, Kumamoto, Japan \\ ${ }^{5}$ Division of Health Sciences, Graduate School of Health Sciences, Kumamoto Health Science University, Kumamoto, Japan
}

The purpose of this study was to investigate the effect of recumbent cycling with integrated volitional control electrical stimulation (IVES) on gait ability in stroke patients. Six stroke patients (all male; average age, $55.7 \pm 8.3$ years) participated. Recumbent cycling (R-cycling) was performed with and without IVES in the power assist (IVES-P) mode. The targeted muscle for electrostimulation was the tibialis anterior. Patients performed 10 min of IVES-P mode plus R-cycling (program A) or R-cycling alone (program B), once per day, 5 times per week. Patients completed two sets of each program, alternating between programs each week. Gait speed and the number of steps numbers on a 10-m walking test was assessed before and after each interventional session. Pro- gram A improved gait speed, but not the number of steps, to a greater extent than that in program B. Specifically, the combined intervention significantly improved gait speed in the first set, but not the second set of the intervention. R-cycling with IVES-P mode improved gait speed during the recovery stage in stroke patients to a greater extent than that achieved with R-cycling alone. Thus, this combined therapy has potential as a standardized treatment in the field of rehabilitation medicine.

Keywords: Recumbent cycling, Electrical stimulation, Gait speed, Stroke patients

\section{INTRODUCTION}

Stroke patients have various symptoms, including spasticity, hemiplegia, imbalance, sensory disturbance, higher brain dysfunction, and so on (Cantagallo et al., 2012; Klingner et al., 2012; Punt and Riddoch, 2006; Wissel et al., 2015). Among these symptoms, hemiplegia especially affects the activities of daily living (ADL) (Fugl-Meyer and Jääskö, 1980). Gait is fundamental in the ADL, and gait ability is a critical factor of the ADL (Mikołajewska, 2013). Thus, for hemiplegia and gait disturbances, various rehabilitative training methods for the affected side have been performed, such as proprioceptive neuromuscular facilitation (Guiu-Tula et al., 2017), electrostimulation (Pomeroy et al., 2006), transcranial magnetic stimulation (Peters et al., 2017), cycling (Van de Port et al., 2007), repetitive standing exercise (Pollock et al., 2014), and gait training with a treadmill (Brauer et al., 2018).

Cycling is often used as a therapeutic exercise for stroke patients (Van de Port et al., 2007). Although the joint movement of the bilateral lower extremities during cycling is different from that during walking, cycling as a rhythmic movement is reciprocally performed; this reciprocal movement is thought to be similar to that in walking. A previous study reported that active cycling exercise reduces the excitability of the paretic vastus medialis $\mathrm{H}$-reflex (Fuchs et al., 2011), while passive cycling activated the unaffected premotor cortex (Peri et al., 2016). Thus, for stroke patients, cycling itself appears to act upon the components of the

\footnotetext{
${ }^{*}$ Corresponding author: Atsushi Doi (iD https://orcid.org/0000-0001-8913-0124 Graduate School of Health Sciences, Kumamoto Health Science University, 325 Izumi-machi, Kumamoto-kitaku, Kumamoto, Japan E-mail: atsushidoi01@gmail.com

Received: October 12, 2018 / Accepted: December 9, 2018
}

This is an Open Access article distributed under the terms of the Creative Commons Attribution Non-Commercial License (http://creativecommons.org/licenses/by-nc/4.0/) which permits unrestricted non-commercial use, distribution, and reproduction in any medium, provided the original work is properly cited. 
central nervous system relevant to gait. However, standard fixed cycling presents a risk of falling for stroke patients with trunk imbalance and motor and sensory hemiplegia. On the other hand, recumbent cycling (R-cycling), which is relatively new, is performed in a semi-supine position. Thus, it is a safer cycling method for patients with imbalance (De Marchis et al., 2015). A program of $30 \mathrm{~min}$ of semirecumbent aerobic cycling appears to help stroke patients regain gait speed (Tang et al., 2009).

Additionally, electrostimulation has been used to recover from muscle weakness and atrophy (Mayr et al., 1999). An advantage of electrostimulation is that it can be utilized in, not only athletes, but also elderly people with disabilities, as the stimulation can be performed in any position, such as supine, side-lying, sitting, and standing (Heidland et al., 2013). A weakness of electrostimulation is that it results in passive muscle contractions, rather than active contractions. However, passive muscle contractions may affect active muscle contractions and contribute to an improvement in the ADL. Recently, a clinical study on integrated volitional control electrical stimulation (IVES), an applied electromyography (EMG)-controlled stimulation system, was published (Yamaguchi et al., 2011). The IVES system, which can potentially stimulate targeted muscle, includes a IVES power assist mode (IVES-P mode) (Yamaguchi et al., 2011). In the IVES-P mode, activation depends on the contraction of the targeted muscle. Namely, if a contraction of the targeted muscle is detected, the system stimulates the muscle at an intensity level proportional to the detected volitional EMG signal. On the other hand, if a targeted muscle contraction is not detected, the system stimulates the muscle at a sub-motor-threshold level (Yamaguchi et al., 2011). Research using the IVES system has mainly focused on the upper extremities (Muraoka et al., 2013; Yamaguchi et al., 2011). However, cycling is often utilized as a therapeutic exercise in stroke patients. Furthermore, research on the combination of cycling and electrostimulation is lacking, especially for IVES and gait training. Therefore, the purpose of this study was to investigate the effect of the combination of IVES and gait training on gait ability in stroke patients.

\section{MATERIALS AND METHODS}

\section{Participants}

Stroke patients admitted to Fukuoka Seisyukai Hospital from 2014 July to 2015 March were invited to participate in this study (Table 1). All six patients were male (average age, $55.7 \pm 8.3$ years) and satisfied the following three conditions: (a) first-onset stroke; (b) able to perform a 10-m independent walking test with or without a cane and short brace; and (c) had a score of over 21 points on the Mini-Mental State Examination. One participant (case 2) quit prior to the end of the experiment because of the discharge from our hospital (at the end of A2, as described below) (Supplementary Figs. 1, 2). All participants provided written informed consent prior to the start of the experiments, in accordance with the Declaration of Helsinki, and the study was approved by the Life Science Committee of Fukuoka Seisyukai Hospital (approval number: 2016-02).

\section{Intervention programs}

The cycling exercise comprised R-cycling (OG Cateye ergociser EC-3500, OG-giken, Tokyo, Japan). The seat was positioned such that there was 10 degrees of knee extension when the patients were fully extended. Patients cycled in the active cycling mode (Fig. 1A). IVES was conducted in the IVES-P mode, and the targeted muscle for electrostimulation was the tibialis anterior (TA) of the affected side. A pair of surface electrodes was used for both recording EMG data (at the TA motor point) and electrical stimulation of the peroneal nerve, which is located under the fibula microcephaly (Fig. 1A, B) (Muraoka, 2002). Patients performed

Table 1. Characteristics of each subject

\begin{tabular}{|c|c|c|c|c|c|c|c|c|c|}
\hline Case No & Age & Gender & Diagnosis & Side & Weeks & Brace & $\begin{array}{c}\text { Initial } \\
\text { ueda-scale (LE) }\end{array}$ & MMSE & $\begin{array}{c}\text { Sensory } \\
\text { (SIAS) }\end{array}$ \\
\hline 1 & 64 & $M$ & Infarction & $\mathrm{L}$ & 8 & Use & 7 & 27 & $2-2$ \\
\hline 2 & 59 & $M$ & Infarction & $\mathrm{R}$ & 4 & No use & 11 & 30 & NP \\
\hline 3 & 50 & $\mathrm{M}$ & Infarction & L & 4 & No use & 12 & 26 & NP \\
\hline 4 & 30 & $M$ & Hemorrhage & L & 1 & No use & 8 & 30 & NP \\
\hline 5 & 55 & $M$ & Infarction & L & 13 & Use & 6 & 30 & NP \\
\hline 6 & 59 & $M$ & Infarction & L & 2 & No use & 8 & 26 & NP \\
\hline
\end{tabular}

Side, paralyzed side (L: left, R: right); Weeks, weeks after stroke onset; Brace, use of the brace for walking test; Initial Ueda-scale (LE), before the programs were started, we evaluated the paralysis of the lower extremities (L/E) on the affected side using the Ueda-scale, which reflects the modified Brunnstrom stage using 12 grades instead of the original 6 grades; MMSE, Mini-Mental State Examination score; Sensory (SIAS), the sensory items in the Stroke Impairment Assessment Set (SIAS); NP, no problem. 


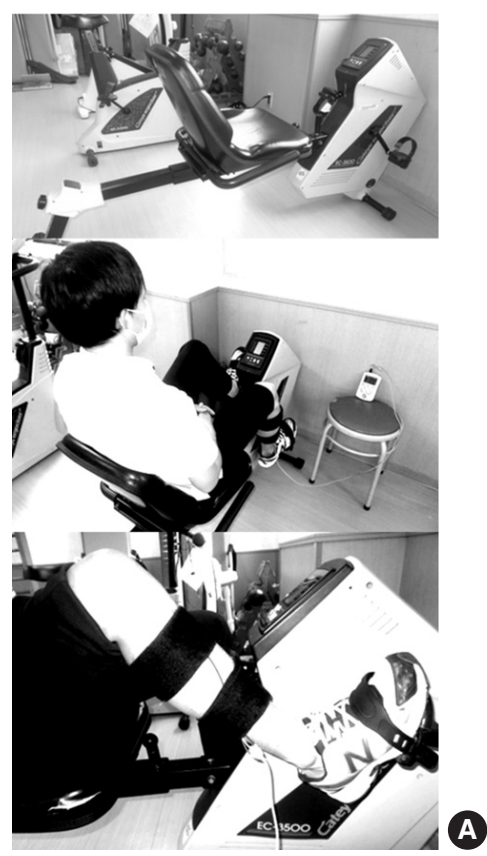

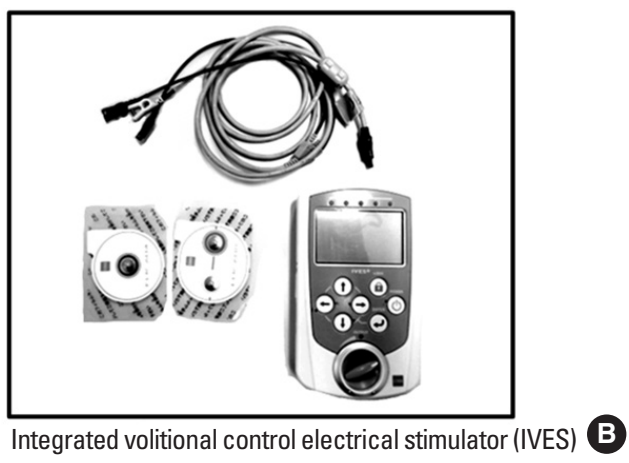

Protocol for intervention

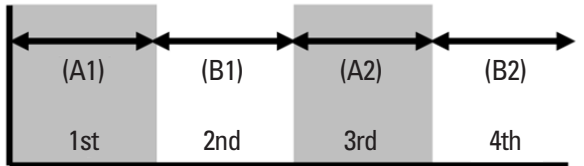

(A1 and A2): R-cycling and IVES-P mode

(B1 and B2): R-cycling

Fig. 1. R-cycling, IVES, and intervention protocol. (A) Upper panel: the R-cycle is shown; Middle panel: a participant is R-cycling; Lower panel: the electrodes of the IVES are shown. (B) The IVES system is shown. (C) The intervention protocol is depicted. IVES, integrated volitional control electrical stimulation; R-cycling, recumbent cycling; $A 1$ and A2, the first and second sets of IVES in the power assist (IVES-P) mode with R-cycling, respectively; B1 and B2, the first and second sets of R-cycling alone, respectively.
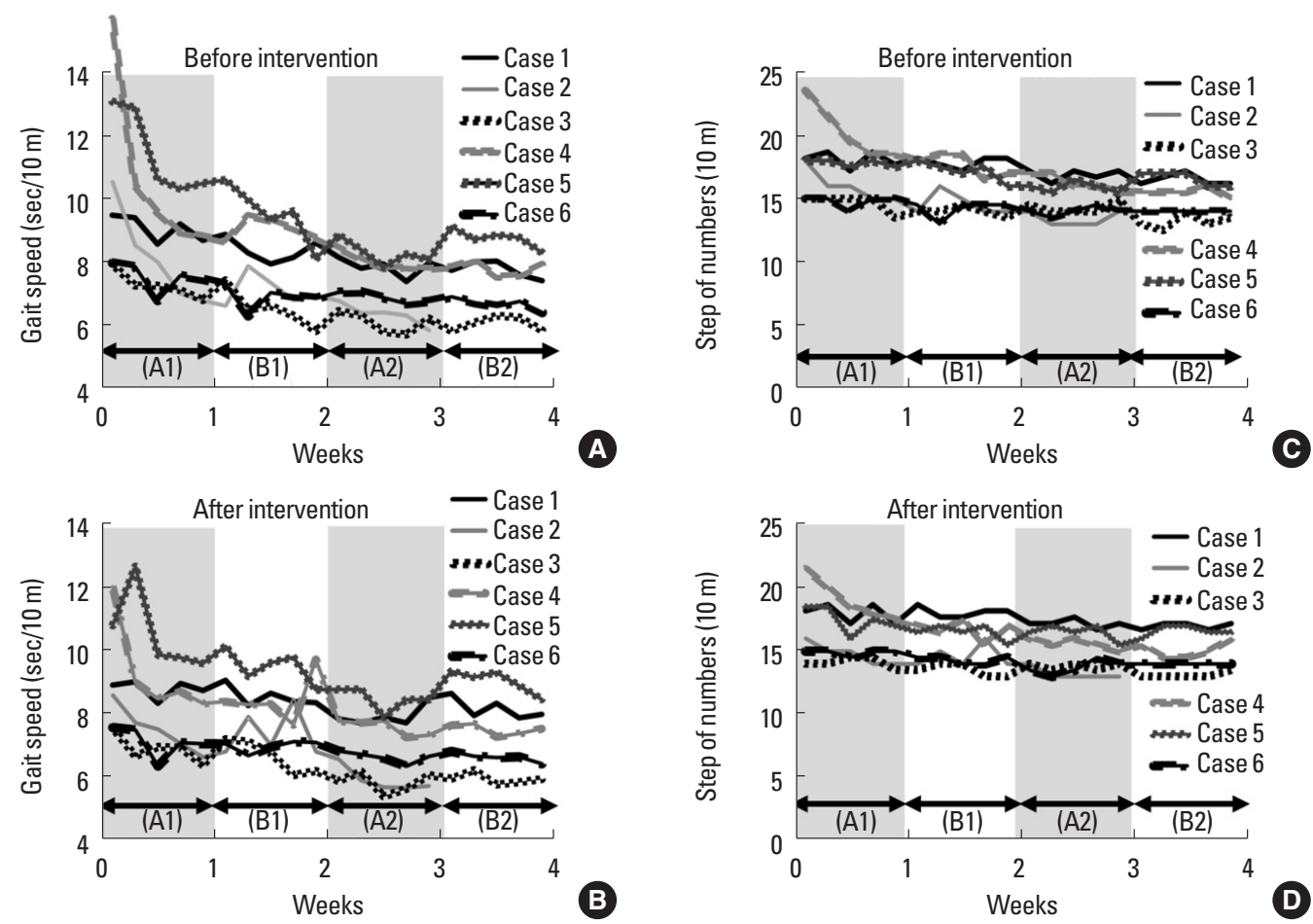

Fig. 2. The gait speed and number of steps on a 10-m walking test before and after the interventions. (A) The gait speed before the intervention. (B) The gait speed after the intervention. (C) The number of steps before the intervention. (D) The number of steps after the intervention. A1 and A2, the first and second sets of IVES-P mode with R-cycling, respectively; B1 and B2, the first and second sets of R-cycling alone, respectively; IVES-P, integrated volitional control electrical stimulation in the power assist. 
10 min of either program A (IVES-P mode plus R-cycling) or program B (only R-cycling) once per day, 5 times per week (Fig. 1C). Patients performed two sets of program $A$ and $B$ ( $A 1$ and $A 2$, and B1 and B2, respectively), alternating between the $A$ and B programs each week (Fig. 1C).

\section{Outcome measures}

Gait speed and the number of steps on a 10-m walking test were assessed before and after each interventional session. For each session, the 10-m test was performed twice and the obtained values were averaged.

\section{Statistical analysis}

Data are expressed as means \pm standard deviation. Gait speed and the number of steps were compared between $\mathrm{A} 1$ and $\mathrm{B} 1$, and between A2 and B2, using two approaches. One analysis focused on the weekly change in gait speed/number of steps for each case (final data minus initial data within each set). The other analysis focused on the average immediate effect (after training minus before the training within each set) for each case. The Wilcoxon signed-rank test was utilized in both analyses. Furthermore, the

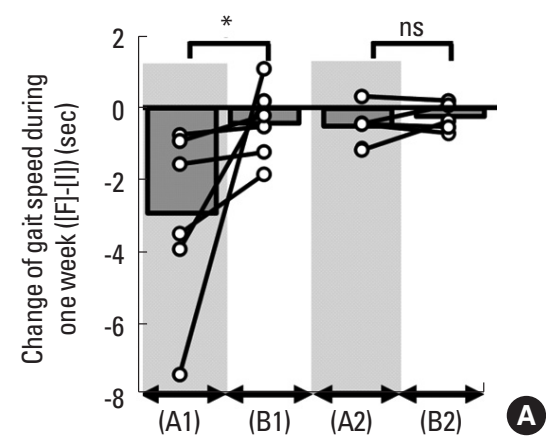

Change of gait speed during 1 week

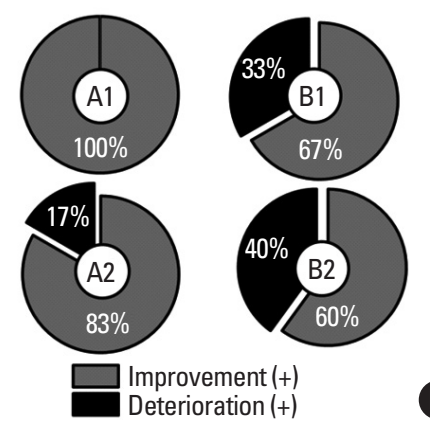

B number of cases showing improvement, deterioration, and no change were compared between programs and sets using the chisquare test. $P$-values of $<0.05$ were considered statistically significant. All statistical analyses were performed using EZR (Saitama Medical Center, Jichi Medical University, Saitama, Japan), which is a graphical user interface for $\mathrm{R}$ (The $\mathrm{R}$ Foundation for Statistical Computing, Vienna, Austria). More precisely, EZR is a modified version of the $\mathrm{R}$ commander, designed to add statistical functions frequently used in biostatistics (Kanda, 2013).

\section{RESULTS}

Overall, the patients showed positive improvements in gait speed and the number of steps for both therapeutic programs (Fig. 3). To better elucidate the changes in these two gait parameters for each case, we have provided the data in multiple arrangements (Supplementary Figs. 1 and 2; Table 2).

\section{R-cycling with IVES-P mode improved gait speed}

The change in gait speed during A1 (IVES-P mode plus R-cycling) was significantly reduced compared to that during B1 (A1:
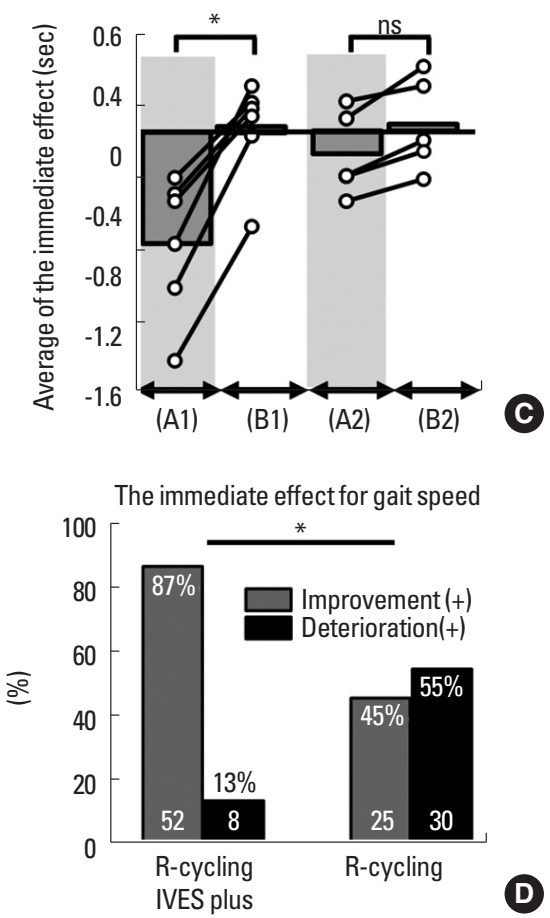

Fig. 3. Changes in gait speed before and after each weekly set. (A) The average weekly change in gait speed is shown ${ }^{*} P<0.05$; not significant [ns]). (B) The weekly changes in gait speed are shown as pie graphs, in terms of improvement and deterioration. (C) The immediate effects of the intervention on gait speed are shown $\left({ }^{*} P<0.05\right.$, ns). The immediate effects of the intervention on gait speed are shown in terms of improvement and deterioration. (D) The immediate effects of the intervention on the gait speed are shown in terms of improvement, and deterioration. A1 and A2, the first and second sets of IVES-P mode with R-cycling, respectively; B1 and B2, the first and second sets of R-cycling alone, respectively; IVES-P, integrated volitional control electrical stimulation in the power assist. 
Table 2. The gait assessments for each subject

\begin{tabular}{|c|c|c|c|c|c|c|c|c|c|c|c|c|}
\hline \multicolumn{13}{|c|}{ Gait speed (sec/10 m) } \\
\hline \multirow{2}{*}{ Case No. } & \multicolumn{3}{|c|}{$\mathrm{A} 1$} & \multicolumn{3}{|c|}{ B1 } & \multicolumn{3}{|c|}{ A2 } & \multicolumn{3}{|c|}{ B2 } \\
\hline & Initial (I) & Final (F) & (FHI) & Initial (I) & Final (F) & (FHI) & Initial (I) & Final (F) & $(F) H I)$ & Initial (I) & Final ( F) & $(\mathrm{FH})$ \\
\hline 1 & 9.52 & 8.77 & -0.75 & 8.91 & 8.39 & -0.52 & 8.18 & 8.55 & 0.37 & 7.77 & 8.02 & 0.25 \\
\hline 2 & 10.58 & 6.66 & -3.92 & 6.65 & 6.84 & 0.19 & 6.81 & 5.75 & -1.06 & - & - & - \\
\hline 3 & 7.97 & 6.41 & -1.56 & 7.45 & 6.23 & -1.22 & 6.48 & 6.09 & -0.39 & 5.85 & 5.97 & 0.12 \\
\hline 4 & 15.75 & 8.37 & -7.38 & 8.68 & 9.76 & 1.08 & 8.48 & 7.36 & -1.12 & 7.88 & 7.56 & -0.32 \\
\hline 5 & 13.13 & 9.64 & -3.49 & 10.64 & 8.8 & -1.84 & 8.89 & 8.49 & -0.4 & 9.16 & 9.5 & -0.66 \\
\hline 6 & 8.01 & 7.09 & -0.92 & 7.36 & 7.13 & -0.13 & 7.08 & 6.69 & -0.39 & 6.94 & 6.46 & -0.48 \\
\hline Avg. & \multicolumn{3}{|c|}{$-3.00 \pm 2.52$} & \multicolumn{3}{|c|}{$-0.42 \pm 1.03$} & \multicolumn{3}{|c|}{$-0.50 \pm 0.54$} & \multicolumn{3}{|c|}{$-0.22 \pm 0.39$} \\
\hline
\end{tabular}

No. of steps $(10 \mathrm{~m})$

\begin{tabular}{|c|c|c|c|c|c|c|c|c|c|c|c|c|}
\hline \multirow{2}{*}{ Case No. } & \multicolumn{3}{|c|}{$\mathrm{A} 1$} & \multicolumn{3}{|c|}{ B1 } & \multicolumn{3}{|c|}{ A2 } & \multicolumn{3}{|c|}{ B2 } \\
\hline & Initial (I) & Final (F) & (FHI) & Initial (I) & Final (F) & (FHI) & Initial (I) & Final (F) & $(F) H I)$ & Initial (I) & Final ( F) & $(F)(I)$ \\
\hline 1 & 18 & 17 & -1 & 18 & 18 & 0 & 17 & 17 & 0 & 16 & 17 & 1 \\
\hline 2 & 18 & 14 & -4 & 14 & 14 & 0 & 14 & 13 & -1 & - & - & - \\
\hline 3 & 15 & 13.5 & -1.5 & 14 & 13 & -1 & 14.5 & 14 & -0.5 & 13 & 13.5 & 0.5 \\
\hline 4 & 23.5 & 17.5 & -6 & 18 & 17 & -1 & 17 & 15 & -2 & 15.5 & 16 & 0.5 \\
\hline 5 & 18 & 17 & -1 & 18 & 15.5 & -2.5 & 16 & 15.5 & -0.5 & 17 & 16.5 & -0.5 \\
\hline 6 & 15 & 15 & 0 & 14.5 & 14.5 & 0 & 14 & 14 & 0 & 14 & 14 & 0 \\
\hline Avg. & \multicolumn{3}{|c|}{$-2.25 \pm 2.27$} & \multicolumn{3}{|c|}{$-0.75 \pm 0.99$} & \multicolumn{3}{|c|}{$-0.67 \pm 0.75$} & \multicolumn{3}{|c|}{$0.30 \pm 0.57$} \\
\hline
\end{tabular}

A1 and A2, the first and second sets of IVES-P mode with R-cycling, respectively; B1 and B2, the first and second sets of R-cycling alone, respectively; Initial (I), at the beginning of the set; Final (F), at the end of the set; (F)-(I), the final value minus the initial value; Avg., the average across subjects.

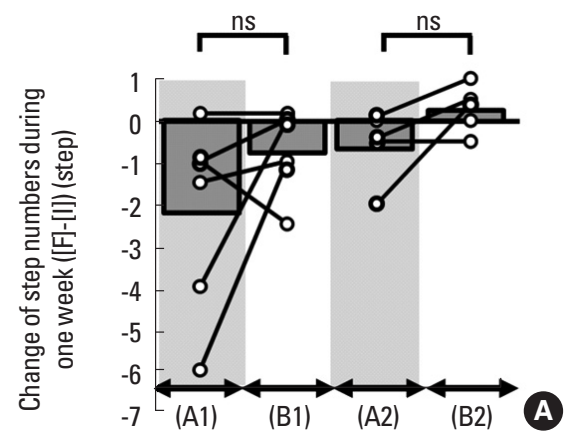

Change of the step numbers during 1 week

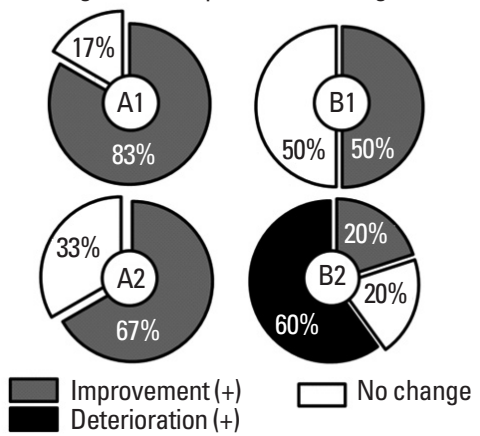

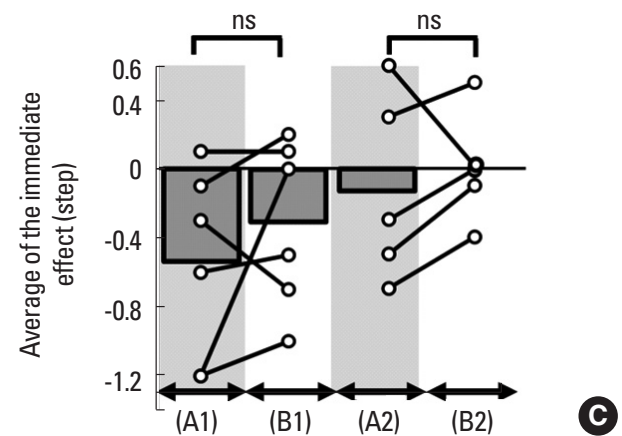

The immediate effect for the step numbers

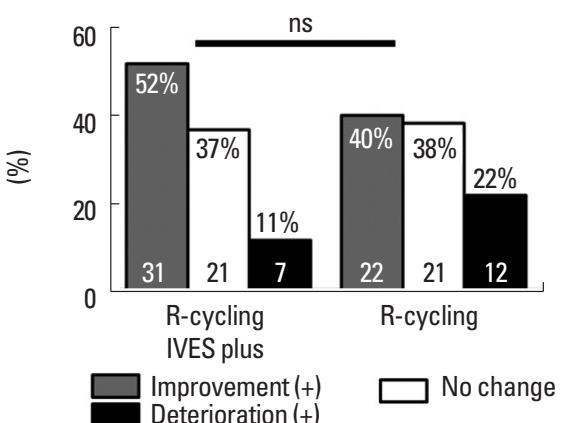

Fig. 4. Change in the number of steps on the 10-m walking test before and after each weekly set. (A) The average weekly change in the number of steps numbers is shown (not significant [ns]). (B) The weekly changes in the number of steps numbers are shown as pie graphs, in terms of improvement, no change, and deterioration. (C) The immediate effects of the intervention on the number of steps are shown (ns). (D) The immediate effects of the intervention on the number of steps are shown in terms of improvement, no change, and deterioration. A1 and A2, the first and second sets of IVES-P mode with R-cycling, respectively; B1 and B2, the first and second sets of R-cycling alone, respectively; IVES-P, integrated volitional control electrical stimulation in the power assist. 
$-3.00 \pm 2.52 \mathrm{sec}, \mathrm{B} 1:-0.42 \pm 1.03 \mathrm{sec}, P<0.05$ ) (Fig. $3 \mathrm{~A}$ ). In contrast, the change in gait speed did not significantly differ between $\mathrm{A} 2$ and B2 (A2: $-0.50 \pm 1.03 \mathrm{sec}, \mathrm{B} 2:-0.22 \pm 0.39 \mathrm{sec} ; P=$ not significant [ns]) (Fig. 3A). The improvement rate in A1, B1, A2, and B2 was $100 \%$ (6 of 6), $67 \%$ ( 4 of 6), $83 \%$ (5 of 6), and $60 \%$ ( 3 of 5), respectively ( $\mathrm{A} 1$ vs. $\mathrm{B} 1$ and $\mathrm{A} 2$ vs. $\mathrm{B} 2, P=$ ns) (Fig. $3 \mathrm{~B}$ ). The immediate effect of therapy on gait speed during $A 1$ was significantly reduced compared to that during B1 (A1: $-0.68 \pm 0.42 \mathrm{sec}$, B1: $0.01 \pm 0.31 \mathrm{sec}, P<0.05$ ) (Fig. 3C). However, the immediate effect of therapy on gait speed did not significantly differ between A2 and B2 (A2: $-0.13 \pm 0.26 \mathrm{sec}, \mathrm{B} 2: 0.05 \pm 0.28 \mathrm{sec}, P=\mathrm{ns}$ ) (Fig. $3 C)$. The rate of a positive effect was much higher for program $A$ (A1 and A2) than for program B (B1 and B2) (improvement and deterioration, program A: $87 \%$ and $13 \%$, respectively; program B: $45 \%$ and $55 \%$, respectively; $P<0.05)$ (Fig. 3D).

\section{No effect of R-cycling with IVES-P mode on the number of steps}

The change in the number of steps on the 10-m walking test during $\mathrm{A} 1$ was not significantly different from that during $\mathrm{B} 1$ (A1: $-2.25 \pm 2.27$, B1: $-0.75 \pm 0.99, P<0.05$ ) (Fig. 4A). Furthermore, change in the number of steps did not significantly differ between A2 and B2 (A2: $-0.67 \pm 0.75, \mathrm{~B} 2: 0.30 \pm 0.57, P=\mathrm{ns})$ (Fig. 4A). The improvement rate in A1, B1, A2, and B2 was $83 \%$ (5 of 6), $50 \%$ (3 of 6), 67\% ( 4 of 6), and 20\% (1 of 5), respectively (A1 vs. B1 and $\mathrm{A} 2$ vs. B2, $P=$ ns) (Fig. $4 \mathrm{~B}$ ). The immediate effect of therapy on the number of steps during $A 1$ was not significantly different from that during B1 (A1: $-0.55 \pm 0.55, \mathrm{~B} 1:-0.31 \pm 0.49$, $P=$ ns) (Fig. 4C). Furthermore, immediate effect of therapy on the number of steps did not significantly differ between A2 and B2 (A2: $-0.13 \pm 0.49, \mathrm{~B} 2:-0.00 \pm 0.32, P=$ ns) (Fig. $4 \mathrm{C}$ ). The rate distribution among improvement, no change, and deterioration was similar for the A and B programs (program A: $52 \%, 37 \%$, and $11 \%$, respectively; program B: $40 \%, 38 \%$, and $22 \%$, respectively; $P=$ ns) (Fig. 4D).

\section{DISCUSSION}

In the present study, IVES-P mode with R-cycling improved the gait speed in stroke patients to a greater extent than that for $\mathrm{R}$-cycling alone. Specifically, the combined intervention significantly improved gait speed during the first set (A1 vs. B1), but not the second set, of the intervention (A2 vs. B2). Furthermore, the combination therapy did not show an effect on the number of steps in the 10-m walking test (over that for R-cycling alone).

\section{The effects of R-cycling on the gait speed and number of} steps

In the first set of R-cycling alone (B1), four out of six patients improved in gait speed (Fig. 3B), and half of the six patients decreased in the number of steps on the 10-m test (Fig. 4B), suggesting that R-cycling by itself contributes to an improvement in gait ability. If $\mathrm{R}$-cycling (B1) was continued for a longer time (i.e., for more than 1 week) and/or with greater frequency, the effects of R-cycling might be more apparent.

\section{The effects of IVES-P mode with R-cycling on the gait speed and number of steps}

It has been reported functional electrical stimulation combined with R-cycling improves aerobic capacity (Aaron et al., 2018), performance on the time up and go test (Alon et al., 2011), motricity index (Ambrosini et al., 2012), and cycling imbalance during exercise (Ambrosini et al., 2012), but not gait speed (Ambrosini et al., 2012), or knee muscle power (Ambrosini et al., 2012). Thus, although adding electrical stimulation with cycling has great effects on "motor activity," this combined therapy does not always have a positive effect on "motor functions," such as gait speed (Ambrosini et al., 2012). To our knowledge, a significant effect of electrical stimulation with cycling on gait speed in such a short intervention period (two 1-week sets) has not been previously reported.

We suggest the following two reasons for the difference in the effect of the combination therapy on gait speed between the first (A1 vs. B1) and second sets (A2 vs. B2). First, the change in gait parameters may reach "zero," suggesting that a plateau phase might occur during the intervention process. Secondly, as the combination therapy was performed first (A1), it may have had an advantage over other trials.

\section{Differences between the results for gait speed and the number of steps, and IVES improvement mechanisms}

We suggest that the observed improvement in gait speed may result from changes in motor function, such as the range of motion (ROM) and muscle activity. For example, an expansion in the hip ROM may affect the step cadence, and muscle activity may influence gait speed. In the present study, we did not measure the step cadence, hip ROM, or muscle activity. However, the number of steps did not differ between intervention programs (programs A and B), suggesting that the improvement in gait speed caused by the combined intervention may result from muscle activity, not from an increase in the step cadence by an expansion of the hip ROM. 
Generally, both the iliopsoas and quadriceps are thought to provide a driving force to advance the lower extremity during the swing phase (Alexander and Schwameder, 2016). In the present study, however, we electrically stimulated the TA (see MATERIALS AND METHODS section); we did not stimulate the iliopsoas or quadriceps. We speculate that TA contraction induced an improvement in gait speed by facilitating quadriceps activity, which is often clinically used as a way to enhance the "quadriceps muscle setting" (Tepperman et al., 1986). We also speculate that TA contraction contributes to the clearance of the swing phase of the leg and accelerates the movement of the leg (Kim et al., 2012). These two factors may efficiently affect gait speed.

The activation of the TA muscle in IVES-P mode, using a biofeedback system, depends on the contraction of the targeted muscle (Yamaguchi et al., 2011), suggesting that active TA contraction and dorsiflexion of the ankle joint is facilitated. This may be the main difference between IVES therapy in the IVES-P mode and traditional functional electrical stimulation, which is simply synchronized to the gait phase and induces a passive muscle contraction (Yamaguchi et al., 2011). Although we did not measure cortical activity, repetitive active TA contractions caused by the IVES-P mode may stimulate, not only the peripheral nervous system (muscle and neuromuscular junction), but also the central nervous system (motoneurons in the spinal ventral horn and the primary motor cortex).

A clinical limitation of the present study is that refined evaluation equipment were lacking. Therefore, we measured gait speed and the numbers of step manually. Recently, several software programs have been developed for video analysis, including the open source software, Kinovea, and so on (Dalal et al., 2018; Damsted et al., 2015). Although it may not always be true that clinical research with advanced equipment is best, we plan to further our research using a video analyzing system to confirm and expand upon the present results.

The advantage of R-cycling as a therapy is that it is easy and safe (De Marchis et al., 2015). Therefore, we strongly believe that for stroke patients, R-cycling is more clinically appropriate than the classical type of cycling, which is usually used in the rehabilitation gym. Currently, some hospitals may not be able to utilize the IVES system, as it is a comparatively new system. However, if the use of the IVES system is expanded worldwide, the combination of safe recumbent cycling with IVES may become a standardized treatment in the field of rehabilitation medicine.

In conclusion, during the recovery stage in stroke patients, R-cycling with IVES improves gait speed, but not the number of steps, to a greater extent than that achieved with R-cycling alone. This combination therapy shows promise in the field of rehabilitation medicine.

\section{CONFLICT OF INTEREST}

No potential conflict of interest relevant to this article was reported.

\section{SUPPLEMENTARY MATERIALS}

Supplementary Figs. 1, 2 can be found via https://doi.org/10. 5213/jer.1836500.250.

\section{REFERENCES}

Aaron SE, Vanderwerker CJ, Embry AE, Newton JH, Lee SCK, Gregory CM. FES-assisted cycling improves aerobic capacity and locomotor function postcerebrovascular accident. Med Sci Sports Exerc 2018;50: 400-406.

Alexander N, Schwameder H. Effect of sloped walking on lower limb muscle forces. Gait Posture 2016;47:62-67.

Alon G, Conroy VM, Donner TW. Intensive training of subjects with chronic hemiparesis on a motorized cycle combined with functional electrical stimulation (FES): a feasibility and safety study. Physiother Res Int 2011;16:81-91.

Ambrosini E, Ferrante S, Ferrigno G, Molteni F, Pedrocchi A. Cycling induced by electrical stimulation improves muscle activation and symmetry during pedaling in hemiparetic patients. IEEE Trans Neural Syst Rehabil Eng 2012;20:320-330.

Brauer SG, Kuys SS, Paratz JD, Ada L. Improving physical activity after stroke via treadmill training and self management (IMPACT): a protocol for a randomised controlled trial. BMC Neurol 2018;18:13.

Cantagallo A, Maini M, Rumiati RI. The cognitive rehabilitation of limb apraxia in patients with stroke. Neuropsychol Rehabil 2012;22:473488.

Dalal KK, Joshua AM, Nayak A, Mithra P, Misri Z, Unnikrishnan B. Effectiveness of prowling with proprioceptive training on knee hyperextension among stroke subjects using videographic observation- a randomised controlled trial. Gait Posture 2018;61:232-237.

Damsted C, Nielsen RO, Larsen LH. Reliability of video-based quantification of the knee- and hip angle at foot strike during running. Int J Sports Phys Ther 2015;10:147-154.

De Marchis C, Ambrosini E, Schmid M, Monticone M, Pedrocchi A, Ferrigno G, D'Alessio T, Conforto S, Ferrante S. Neuro-mechanics of mus- 
cle coordination during recumbent pedaling in post-acute stroke patients. Conf Proc IEEE Eng Med Biol Soc 2015;2015:246-249.

Fuchs DP, Sanghvi N, Wieser J, Schindler-Ivens S. Pedaling alters the excitability and modulation of vastus medialis H-reflexes after stroke. Clin Neurophysiol 2011;122:2036-2043.

Fugl-Meyer AR, Jääskö L. Post-stroke hemiplegia and ADL-performance. Scand J Rehabil Med Suppl 1980;7:140-152.

Guiu-Tula FX, Cabanas-Valdés R, Sitjà-Rabert M, Urrútia G, Gómara-Toldrà N. The Efficacy of the proprioceptive neuromuscular facilitation (PNF) approach in stroke rehabilitation to improve basic activities of daily living and quality of life: a systematic review and meta-analysis protocol. BMJ Open 2017;7:e016739.

Heidland A, Fazeli G, Klassen A, Sebekova K, Hennemann H, Bahner U, Di Iorio B. Neuromuscular electrostimulation techniques: historical aspects and current possibilities in treatment of pain and muscle waisting. Clin Nephrol 2013;79 Suppl 1:S12-23.

Kanda Y. Investigation of the freely available easy-to-use software 'EZR' for medical statistics. Bone Marrow Transplant 2013;48:452-458.

Kim JH, Chung Y, Kim Y, Hwang S. Functional electrical stimulation applied to gluteus medius and tibialis anterior corresponding gait cycle for stroke. Gait Posture 2012;36:65-67.

Klingner CM, Witte OW, Günther A. Sensory syndromes. Front Neurol Neurosci 2012;30:4-8.

Mayr W, Bijak M, Girsch W, Hofer C, Lanmüller H, Rafolt D, Rakos M, Sauermann S, Schmutterer C, Schnetz G, Unger E, Freilinger G. MYOSTIM-FES to prevent muscle atrophy in microgravity and bed rest: preliminary report. Artif Organs 1999;23:428-431.

Mikołajewska E. Associations between results of post-stroke NDT-Bobath rehabilitation in gait parameters, ADL and hand functions. Adv Clin Exp Med 2013;22:731-738.

Muraoka Y. Development of an EMG recording device from stimulation electrodes for functional electrical stimulation. Front Med Biol Eng 2002;11:323-333.

Muraoka Y, Tanabe S, Yamaguchi T, Takeda K. Specifications of an electromyogram-driven neuromuscular stimulator for upper limb func- tional recovery. Conf Proc IEEE Eng Med Biol Soc 2013;2013:277-280.

Peri E, Ambrosini E, Pedrocchi A, Ferrigno G, Nava C, Longoni V, Monticone M, Ferrante S. Can FES-augmented active cycling training improve locomotion in post-acute elderly stroke patients? Eur J Transl Myol 2016;26:6063.

Peters HT, Dunning K, Belagaje S, Kissela BM, Ying J, Laine J, Page SJ. Navigated transcranial magnetic stimulation: a biologically based assay of lower extremity impairment and gait velocity. Neural Plast 2017;2017:6971206.

Pollock A, Gray C, Culham E, Durward BR, Langhorne P. Interventions for improving sit-to-stand ability following stroke. Cochrane Database Syst Rev 2014;(5):CD007232.

Pomeroy VM, King L, Pollock A, Baily-Hallam A, Langhorne P. Electrostimulation for promoting recovery of movement or functional ability after stroke. Cochrane Database Syst Rev 2006;(2):CD003241.

Punt TD, Riddoch MJ. Motor neglect: implications for movement and rehabilitation following stroke. Disabil Rehabil 2006;28:857-864.

Tang A, Sibley KM, Thomas SG, Bayley MT, Richardson D, Mcllroy WE, Brooks D. Effects of an aerobic exercise program on aerobic capacity, spatiotemporal gait parameters, and functional capacity in subacute stroke. Neurorehabil Neural Repair 2009;23:398-406.

Tepperman PS, Mazliah J, Naumann S, Delmore T. Effect of ankle position on isometric quadriceps strengthening. Am J Phys Med 1986;65: 69-74.

van de Port IG, Wood-Dauphinee S, Lindeman E, Kwakkel G. Effects of exercise training programs on walking competency after stroke: a systematic review. Am J Phys Med Rehabil 2007;86:935-951.

Wissel J, Verrier M, Simpson DM, Charles D, Guinto P, Papapetropoulos S, Sunnerhagen KS. Post-stroke spasticity: predictors of early development and considerations for therapeutic intervention. PM R 2015;7:6067.

Yamaguchi T, Tanabe S, Muraoka Y, Imai S, Masakado Y, Hase K, Kimura A, Liu M. Effects of integrated volitional control electrical stimulation (IVES) on upper extremity function in chronic stroke. Keio J Med 2011;60:90-95. 